19,11

\title{
Термодинамические свойства оксипентафторида ванадия (IV), $\left(\mathrm{NH}_{4}\right)_{3} \mathrm{VOF}_{5}$
}

\author{
(C) Е.В. Богданов ${ }^{1,2}$, Е.И. Погорельцев ${ }^{1,3}$, А.В. Карташев ${ }^{1}$, М.В. Горев ${ }^{1,3}$, М.С. Молокеев ${ }^{1,3}$, \\ С.В. Мельникова ${ }^{1}$, И.Н. Флеров ${ }^{1,3}$, Н.М. Лапташ ${ }^{4}$ \\ ${ }^{1}$ Институт фризики им. Л.В. Киренского СО РАН ФИЦ КНЦ СО РАН, \\ Красноярск, Россия \\ ${ }^{2}$ Институт инженерных систем и энергетики, Красноярский аграрный университет, \\ Красноярск, Россия \\ ${ }^{3}$ Институт инженерной фризики и радиоэлектроники, Сибирский федеральный университет, \\ Красноярск, Россия \\ ${ }^{4}$ Институт химии ДО РАН. Владивосток, Россия \\ E-mail: evbogdanov@iph.krasn.ru
}

Поступила в Редакцию 22 января 2020 г.

В окончательной редакции 30 января 2020 г.

Принята к публикации 30 января 2020 г.

Выращены кристаллы $\left(\mathrm{NH}_{4}\right)_{3} \mathrm{VOF}_{5}$, однородность и однофазность которых установлена рентгеновскими методами (энергодисперсионной спектроскопи, рентгеновской фотоэлектронной спектроскопии и рентгеновской дифракции). Проведенные исследования температурных зависимостей теплоемкости, энтропии, деформации и барической восприимчивости свидетельствуют о наличии в кристаллах $\left(\mathrm{NH}_{4}\right)_{3} \mathrm{VOF}_{5}$ трех фазовых переходов, связанных со структурными превращениями. Построенная фазовая $T-p$ диаграмма показывает температурные границы устойчивости реализующихся кристаллических фаз в $\left(\mathrm{NH}_{4}\right)_{3} \mathrm{VOF}_{5}$. Оптические и диэлектрические исследования свидетельствуют о сегнетоэластической природе фазовых переходов. Анализ экспериментальных данных совместно с данными изоструктурного кристалла $\left(\mathrm{NH}_{4}\right)_{3} \mathrm{VO}_{2} \mathrm{~F}_{4}$ позволяет выделить особенности физических свойств оксифторидов, содержащих ванадий различной валентности (IV и V).

Ключевые слова: оксифториды, фазовые переходы, теплоемкость, двупреломление, тепловое расширение, восприимчивость к давлению.

DOI: $10.21883 /$ FTT.2020.07.49485.008

\section{1. Введение}

Структура комплексных фторидов и оксифторидов на основе ванадия $A_{2} A^{\prime} \mathrm{VO}_{x} \mathrm{~F}_{6-x}\left(A, A^{\prime}: \mathrm{NH}_{4}, \mathrm{Cs}, \mathrm{Rb}, \mathrm{K}\right.$, $\mathrm{Na} ; x=0,1,2)$ может быть образована анионными полиэдрами разной симметрии, вследствие способности центрального атома устойчиво существовать в различных валентных состояниях [1]. В отличие от фторидов, в которых шестикоординированный полиэдр $\left[\mathrm{VF}_{6}\right]^{3-}$ представляет собой правильный октаэдр [2], в соединениях с $x=1$ и 2 его локальная симметрия является соответственно тетрагональной и ромбической, что приводит к появлению локального полярного момента [3,4]. Однако, несмотря на последнее обстоятельство, кристаллическая структура большинства соединений $A_{2} A^{\prime} \mathrm{VO}_{2} \mathrm{~F}_{4}$ и $A_{2} A^{\prime} \mathrm{VOF}_{5}$ является высокосимметричной (пр.гр. $F m \overline{3} m$, $Z=4)$, что обусловлено, как минимум, разупорядочением фторкислородных лигандов $[5,6]$.

B аммонийных оксифторидах $\left(\mathrm{NH}_{4}\right)_{3} \mathrm{VOF}_{5}$ и $\left(\mathrm{NH}_{4}\right)_{3} \mathrm{VO}_{2} \mathrm{~F}_{4}$ лиганды распределены одинаковым образом по двум кристаллографическим позициям $24 e+96 j$, однако анионные полиэдры $\left[\mathrm{VO}_{2} \mathrm{~F}_{4}\right]^{3-}$ и $\left[\mathrm{VOF}_{5}\right]^{3-}$ характеризуются разным числом пространственных ориентаций: соответственно двенадцать и шесть [6]. Результаты исследований методом колебательной спектроскопии указывают на динамический характер ориентационного разупорядочения анионных групп, который более выражен в случае $\left(\mathrm{NH}_{4}\right)_{3} \mathrm{VOF}_{5}$ [7]. Аммонийные группы также оказываются разупорядоченными. Тетраэдр в позиции $4 b$ имеет восемь пространственных ориентаций из-за разупорядочения атомов водорода по позициям $96 j$, а два тетраэдра в положении $8 c$ разупорядочены по положениям $32 f$ и характеризуются четырьмя пространственными ориентациями.

В соответствии с данными оптических, теплофизических и диэлектрических исследований кристалл $\left(\mathrm{NH}_{4}\right)_{3} \mathrm{VO}_{2} \mathrm{~F}_{4}$ претерпевает последовательность из четырех фазовых переходов при температурах $T_{1}=438 \mathrm{~K}$, $T_{2}=244 \mathrm{~K}, T_{3}=210 \mathrm{~K}$ и $T_{4}=205 \mathrm{~K}$, которые сопровождаются изменением симметрии $F m \overline{3} m \leftrightarrow \operatorname{Immm}(I 222) \leftrightarrow$ ромбическая $\leftrightarrow P 112 / m \leftrightarrow P \overline{1}[8,9]$. Калориметрические измерения позволили определить энергетические характеристики переходов, позволяющие анализировать механизмы структурных искажений. Суммарное изменение энтропии для последовательности фазовых переходов в кристалле $\left(\mathrm{NH}_{4}\right)_{3} \mathrm{VO}_{2} \mathrm{~F}_{4}\left(\sum_{i} \Delta S_{i} \approx 17 \mathrm{~J} / \mathrm{mol} \cdot \mathrm{K}\right)$ оказалось значительно меньше величины энтропии, которая следует из модели разупорядочения структурных элементов в фазе $F m \overline{3} m\left(\sum_{i} \Delta S_{i} \approx 34 \mathrm{~J} / \mathrm{mol} \cdot \mathrm{K}\right)$ [6]. Существенная роль аммонийного катиона в механизмах 
структурных искажений установлена при исследовании дейтерированного кристалла $\left(\mathrm{ND}_{4}\right)_{3} \mathrm{VO}_{2} \mathrm{~F}_{4}$ [10]. Дейтерирование привело к изменению химического давления и, как следствие, к изменению температур фазовых переходов и уменьшению ангармонизма колебаний тетраэдрических катионов, приведшему к уменьшению величины суммарной энтропии $\left(\sum_{i} \Delta S_{i} \approx 15 \mathrm{~J} / \mathrm{mol} \cdot \mathrm{K}\right)$ по сравнению с протонированным кристаллом $\left(\mathrm{NH}_{4}\right)_{3} \mathrm{VO}_{2} \mathrm{~F}_{4}$ [9].

В кристаллах $\left(\mathrm{NH}_{4}\right)_{3} \mathrm{VOF}_{5}$, согласно поисковым исследованиям, наблюдается последовательность из трех фазовых превращений $F m \overline{3} m \leftrightarrow I m m m \leftrightarrow ? \leftrightarrow$ ? при температурах $T_{1}=349 \mathrm{~K}, \quad T_{2}=230 \mathrm{~K}$ и $T_{3}=221 \mathrm{~K} \quad[6]$. Несмотря на подробные исследования структуры в фазах $F m \overline{3} m$ и Immm, информация обоих физических свойствах отсутствует, что затрудняет анализ природы и механизма фазовых переходов.

В настоящей работе выполнены исследования оптических, теплофизических, структурных и диэлектрических свойств кристалла $\left(\mathrm{NH}_{4}\right)_{3} \mathrm{VOF}_{5}$ в широком интервале температур и давлений. Установлен характер влияния внешнего гидростатического и химического давлений на устойчивость реализующихся искаженных фаз.

\section{2. Экспериментальные методы и результаты исследований}

Получение исходных кристаллов оксофторованадатов аммония выполнено при помощи механохимического взаимодействия исходных оксидов ванадия (или ванадатов) с гидродифторидом аммония $\left(\mathrm{NH}_{4} \mathrm{HF}_{2}\right.$, температура плавления $T_{\text {melt }}=126^{\circ} \mathrm{C}$ ). [6]. В результате реакции при растирании компонентов при обычных условиях были получены исходные комплексы $\left(\mathrm{NH}_{4}\right)_{3} \mathrm{VO}_{2} \mathrm{~F}_{4}$ и $\left(\mathrm{NH}_{4}\right)_{3} \mathrm{VF}_{6}$ :

$$
\begin{gathered}
\mathrm{NH}_{4} \mathrm{VO}_{3}+2 \cdot \mathrm{NH}_{4} \mathrm{HF}_{2}=\left(\mathrm{NH}_{4}\right)_{3} \mathrm{VO}_{2} \mathrm{~F}_{4}+\mathrm{H}_{2} \mathrm{O} \\
\mathrm{VF}_{3}+3 \cdot \mathrm{NH}_{4} \mathrm{HF}_{2}=\left(\mathrm{NH}_{4}\right)_{3} \mathrm{VF}_{6}+3 \cdot \mathrm{HF} .
\end{gathered}
$$

При дальнейшем растворении стехиометрических количеств полученных комплексов в воде при подогреве на водяной бане и последующем медленном выпаривании зеленовато-голубого раствора кристаллизовались хорошо ограненные октаэдры $\left(\mathrm{NH}_{4}\right)_{3} \mathrm{VOF}_{5}$ ярко-голубого цвета:

$$
\left(\mathrm{NH}_{4}\right)_{3} \mathrm{VO}_{2} \mathrm{~F}_{4}+\left(\mathrm{NH}_{4}\right)_{3} \mathrm{VF}_{6}=2 \cdot\left(\mathrm{NH}_{4}\right)_{3} \mathrm{VOF}_{5} \text {. }
$$

Анализ методом энергодисперсионной рентгеновской спектроскопии, выполненный на электронном сканирующем микроскопе высокого разрешения Hitachi 5500 с энергодисперсионной приставкой EDS Thermo Scientific, подтвердил состав монокристаллов $\left(\mathrm{NH}_{4}\right)_{3} \mathrm{VOF}_{5}$, давая соотношение $\mathrm{O}: \mathrm{F}$, близкое к $1: 5$.

Полученные кристаллы были исследованы методом рентгеновской фотоэлектронной спектроскопии. Из

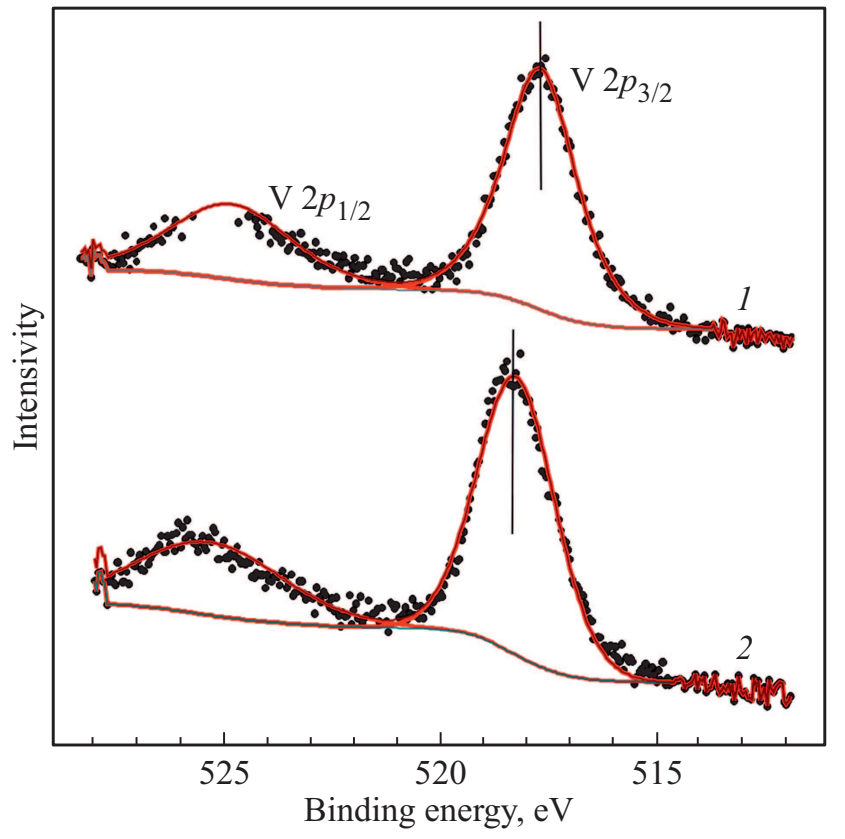

Рис. 1. Спектры рентгеновской фотоэмиссии для $\left(\mathrm{NH}_{4}\right)_{3} \mathrm{VOF}_{5}(1)$ и $\left(\mathrm{NH}_{4}\right)_{3} \mathrm{VO}_{2} \mathrm{~F}_{4}(2)$.

спектров рентгеновской фотоэмиссии получена информация о природе химической связи изучаемых элементов. Рассчитанные из спектров значения энергии связи (линия $\left.\mathrm{V} 2 \mathrm{p}_{3 / 2}\right)$ для исходного $\left(\mathrm{NH}_{4}\right)_{3} \mathrm{VO}_{2} \mathrm{~F}_{4}$ и исследуемого $\left(\mathrm{NH}_{4}\right)_{3} \mathrm{VOF}_{5}$ кристаллов, составили, соответственно $E_{b}=518.3 \mathrm{eV}$ и $E_{b}=517.7 \mathrm{eV}$ (рис. 1 ). Значительное различие в величинах энергии связи $\Delta E_{b}=0.6 \mathrm{eV}$ позволяет однозначно идентифицировать ванадиевые соединения. Спектры также показывают, что в выращенных кристаллах $\left(\mathrm{NH}_{4}\right)_{3} \mathrm{VOF}_{5}$ ванадий присутствует только в одной форме $\mathrm{V}^{4+}$.

Данные о структуре $\left(\mathrm{NH}_{4}\right)_{3} \mathrm{VOF}_{5}$ получены методом рентгеновской дифракции при помощи порошкового дифрактометра Bruker D8 ADVANCE (излучение линии $\left.K_{\alpha} \mathrm{Cu}\right)$ и линейного детектора VANTEC $\left(2 \theta \approx 0.016^{\circ}\right.$, $t \approx 0.2 \mathrm{~s} / 2 \theta)$. Исследования выполнены в температурном диапазоне $133-423 \mathrm{~K}$ с шагом $\sim 10 \mathrm{~K}$ при помощи температурной камеры ТTK450 Anton Paar.

Уточнение модели структуры высокотемпературной фазы проводилось методом Ритвельда (рис. 2) с использованием программы TOPAS 4.2 [11]. Индицирование рентгенограмм, полученных в интервале температур $353-423 \mathrm{~K}$, показало, что элементарная ядейка является кубической гранецентрированной (пр. гр. $F m \overline{3} m, Z=4$, см. табл. 1), дополнительных рефлексов не наблюдалось.

При понижении температуры происходит расщепление основных рефлексов кубической фазы (рис. 3), которое, согласно методу гомологии [12], указывает на появление при комнатной температуре ромбической структуры. Ниже температуры фазового перехода $T_{1}=349 \mathrm{~K}$ наблюдаются сверхструктурные рефлексы, соответствующие нестабильности в точке $k=(2 / 3,2 / 3,0)$ зоны 


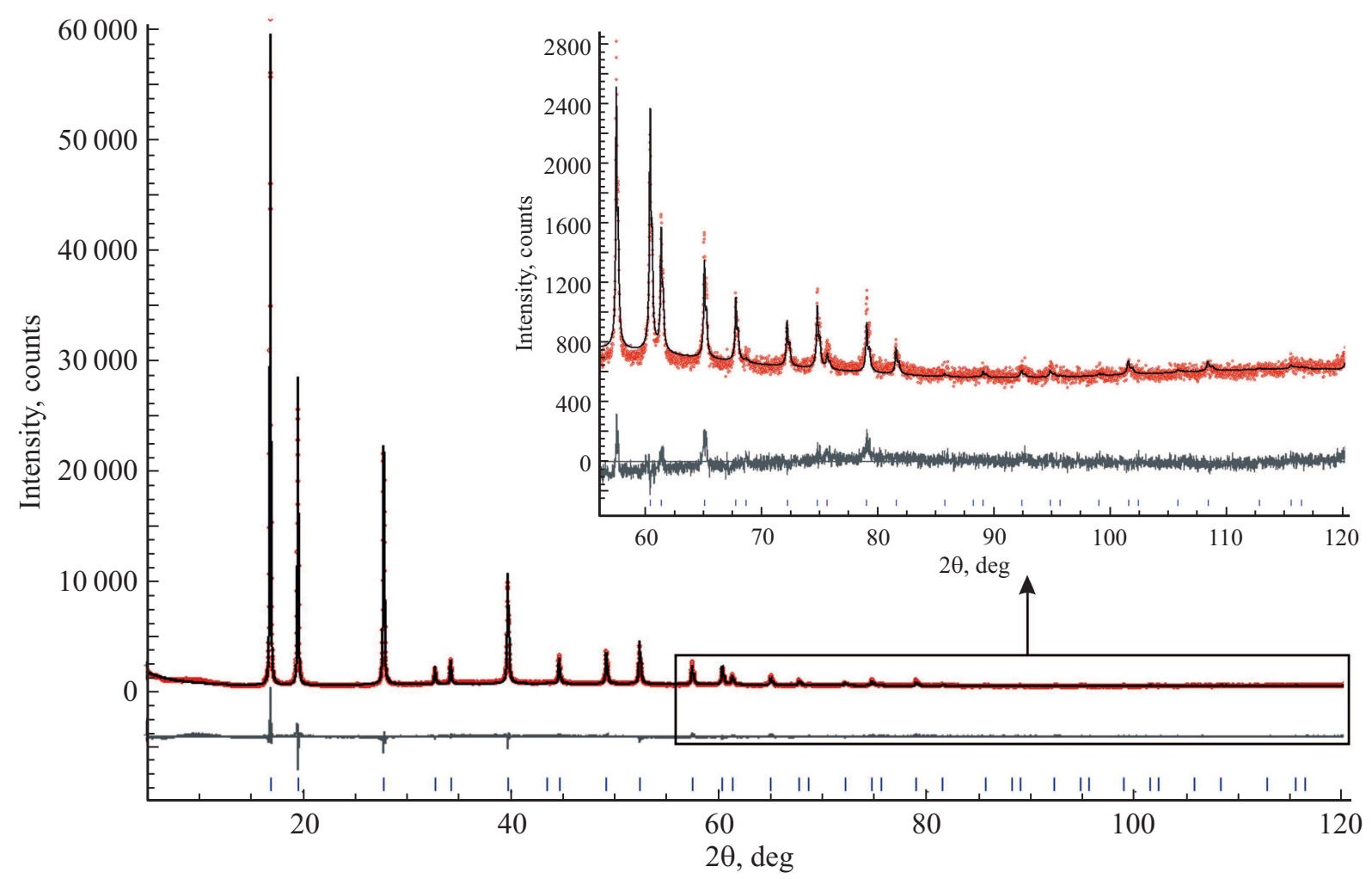

Рис. 2. Разностная рентгенограмма Ритвельда, полученная при уточнении кристаллической структуры $\left(\mathrm{NH}_{4}\right)_{3} \mathrm{VOF}_{5}$ в кубической фазе $(T=383 \mathrm{~K})$.

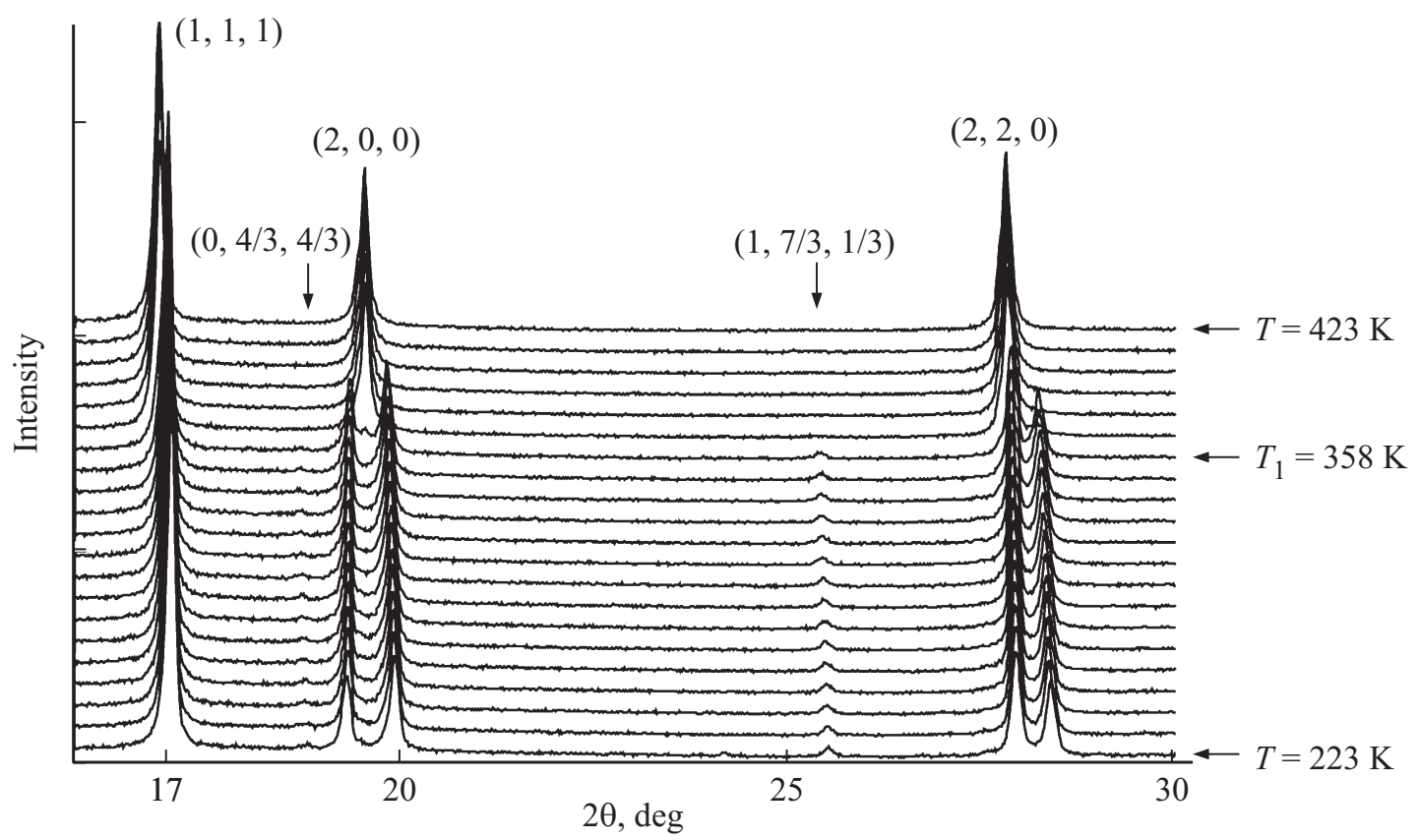

Рис. 3. Фрагмент рентгенограмм $\left(\mathrm{NH}_{4}\right)_{3} \mathrm{VOF}_{5}$ при температуре $T$, меняющейся в диапазоне $223-423 \mathrm{~K}$ с шагом $\Delta T=10 \mathrm{~K}$, в скобках указаны индексы кубической ячейки.

Бриллюэна, что говорит о существенном увеличении объема примитивной ячейки, минимум в три раза. Индицирование и уточнение профиля рентгенограммы показало, что элементарная ячейка описывает- ся пространственной группой Immm: $a=9.1685(2) \AA$, $b=18.9112(8) \AA, c=6.3014(3) \AA$. Наблюдается существенное изменение параметров ромбической ячейки по сравнению с кубической ячейкой: $a_{\text {orth }}=a_{c u b}$; 
Таблица 1. Основные параметры уточнения структуры кристалла $\left(\mathrm{NH}_{4}\right)_{3} \mathrm{VOF}_{5}$, пр. гр. $F m \overline{3} m: a$ и $V$ - параметр и объем элементарной ячейки, $Z$ - число формульных единиц в ячейке, $M_{r}$ - относительный молекулярный вес формульной единицы, $2 \theta$ - интервал углов рентгенограммы; факторы достоверности: $R_{p}-$ профильный, $R_{w p}-$ весовой профильный, $R_{B}-$ брэгговский, $\chi^{2}-$ качество подгонки $R_{w p} / R_{\exp }$, где $R_{\exp }-$ ожидаемый профильный фактор.

\begin{tabular}{c|c}
\hline Параметр & Значение \\
\hline$a, \AA$ & $9.0710(2)$ \\
$V, \AA^{3}$ & $746.38(4)$ \\
$Z$ & 4 \\
$M_{r}$ & 216.05 \\
$2 \theta$ & $5-120$ \\
$R_{w p}, \%$ & 7.677 \\
$R_{p}, \%$ & 6.089 \\
$R_{\text {exp }} \%$ & 3.292 \\
$\chi^{2}$ & 1.11 \\
$R_{B}, \%$ & 2.87
\end{tabular}

$b_{\text {orth }}=3 b_{\text {cub }} / 2+3 c_{c u b} / 2 ; \quad c_{\text {orth }}=b_{c u b} / 2-c_{c u b} / 2, \quad$ где $a_{\text {orth }}, b_{\text {orth }}, c_{\text {orth }}$ - базисные векторы ромбической ячейки, $a_{c u b}, b_{c u b}, c_{c u b}-$ базисные векторы кубической ячейки (рис. 4).

При дальнейшем понижении температуры происходит незначительное расщепление главных рефлексов, которое может быть связано с наличием второго или даже нескольких фазовых переходов. При этом кристаллическая ячейка становится либо моноклинной, либо триклинной. Анализ сверхструктурных рефлексов показал, что они соответствуют появлению нестабильности в точках $k=(1 / 2,1 / 2,1 / 2)$ и $k=(1 / 2,0,0)$ зоны Бриллюэна. Минимальный объем примитивной ячейки при этом становится в двенадцать раз больше объема примитивной кубической ячейки и установление параметров ячеек такой искаженной фазы затруднено.

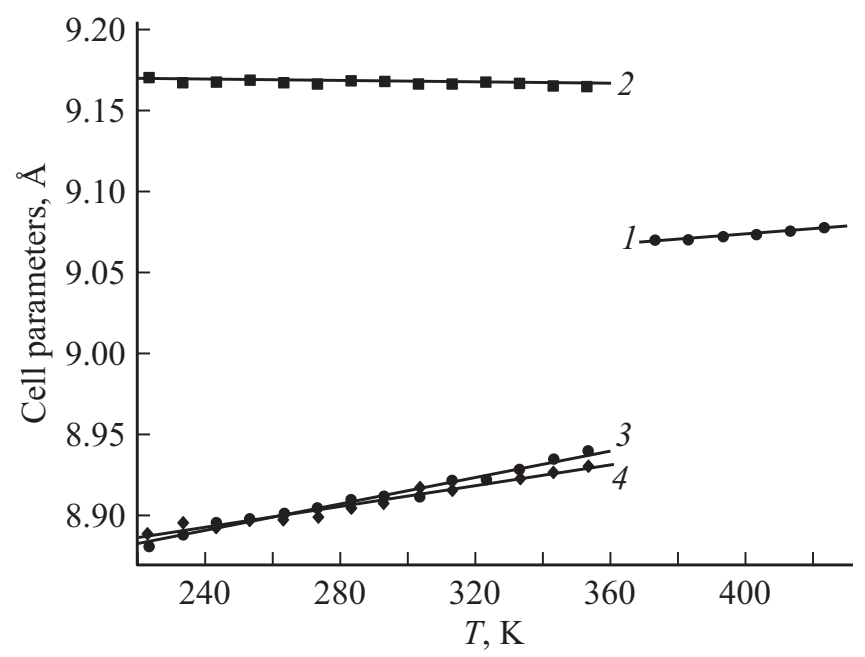

Рис. 4. Температурные зависимости параметров ячеек $\left(\mathrm{NH}_{4}\right)_{3} \mathrm{VOF}_{5}: a_{\text {cub }}(1), a_{\text {otrh }}(2) ; b_{\text {orth }} \sqrt{2} / 3$ (3) и $c_{\text {orth }} \sqrt{2}(4)$.
Оптические исследования были выполнены при помощи поляризационного микроскопа Axioskop-40 и температурной камеры Linkam LTS 350. Температурные исследования проводились в квазистатическом режиме с точностью $\pm 0.1 \mathrm{~K}$ в температурном диапазоне $100-400 \mathrm{~K}$. Положение погасания и угол поворота индикатрисы были определены с точностью $\sim 0.5^{\circ}$. Двупреломление измерялось с помощью компенсатора Berek (Leica) c точностью $\sim 0.00001$.

Для исследований были отобраны тонкие ростовые пластинки $\left(\mathrm{NH}_{4}\right)_{3} \mathrm{VOF}_{5}$ (толщиной $\left.30-100 \mu \mathrm{m}\right)$, которые имели ориентацию в плоскостях (111) и (110) кубической кристаллической структуры. В высокотемпературной области образец $\left(\mathrm{NH}_{4}\right)_{3} \mathrm{VOF}_{5}$ является оптически изотропным, $\Delta n=0$ (рис. $5, a$ и $5, b$ ), что подтверждает исходную кубическую симметрию кристалла.

Ниже температуры $350 \mathrm{~K}$ в поле зрения микроскопа появляется двойниковая картина (рис. 5,c) в виде широких полос с границами, расположенными под углом $120^{\circ}$, что указывает на потерю кристаллом оси третьего порядка в процессе охлаждения при температуре $T_{1 \downarrow}$. Прямое погасание образовавшихся двойников вдоль [110] также подтверждает реализацию ромбической симметрии при комнатной температуре.

Фазовый переход сопровождается скачкообразным появлением оптической анизотропии (рис. 5, $a$ ). В процессе нагревания значение температуры фазового перехода $T_{1 \uparrow}$ зависит от скорости нагрева и может изменяться в интервале 349-357 K, тогда как при охлаждении температура $T_{1 \downarrow}$ практически постоянна и равна $338 \mathrm{~K}$. Измерения $\Delta n(T)$ в квазистатическом режиме с шагом $\Delta T \approx 0.1 \mathrm{~K}$ позволили определить равновесные температуры фазовых переходов $T_{1 \uparrow}=357.1 \mathrm{~K}$ и $T_{1 \downarrow}=338.1 \mathrm{~K}$. Значительный гистерезис $\delta T_{1} \approx 19 \mathrm{~K}$ однозначно свидетельствует, что высокотемпературный фазовый переход является превращением первого рода.

При охлаждении ниже температуры $230 \mathrm{~K}$ двойниковая картина значительно усложняется, а погасание образца полностью исчезает (рис. $5, d$ ). При первом охлаждении наблюдается аномалия $\Delta n(T)$ при температуре $T_{3 \downarrow} \approx 218 \mathrm{~K}$, это сопровождается появлением дополнительного двойникования кристалла. В процессе нагревания изменение $\Delta n(T)$ происходит при более высокой температуре $T_{2 \uparrow}=228 \mathrm{~K}$, это значение воспроизводится при дальнейших нагревах/охлаждениях образца.

Отсутствие погасания образцов при низких температурах свидетельствует о самой низкой группе симметрии кристалла $P \overline{1}$ или $P 1$ в этой фазе, а ступенчатое аномальное поведение двупреломления (рис. $5, a$ ) говорит о наличии некоторой промежуточной (моноклинной) фазы.

Для получения сведений о количестве, температуре и интегральных энергетических характеристиках фазовых переходов в кристаллах $\left(\mathrm{NH}_{4}\right)_{3} \mathrm{VOF}_{5}$ были выполнены исследования температурной зависимости теплоемкости $C_{p}(T)$ методом адиабатической калориметрии. Исследуемый образец с общей массой $\sim 1.1 \mathrm{~g}$ герметично 

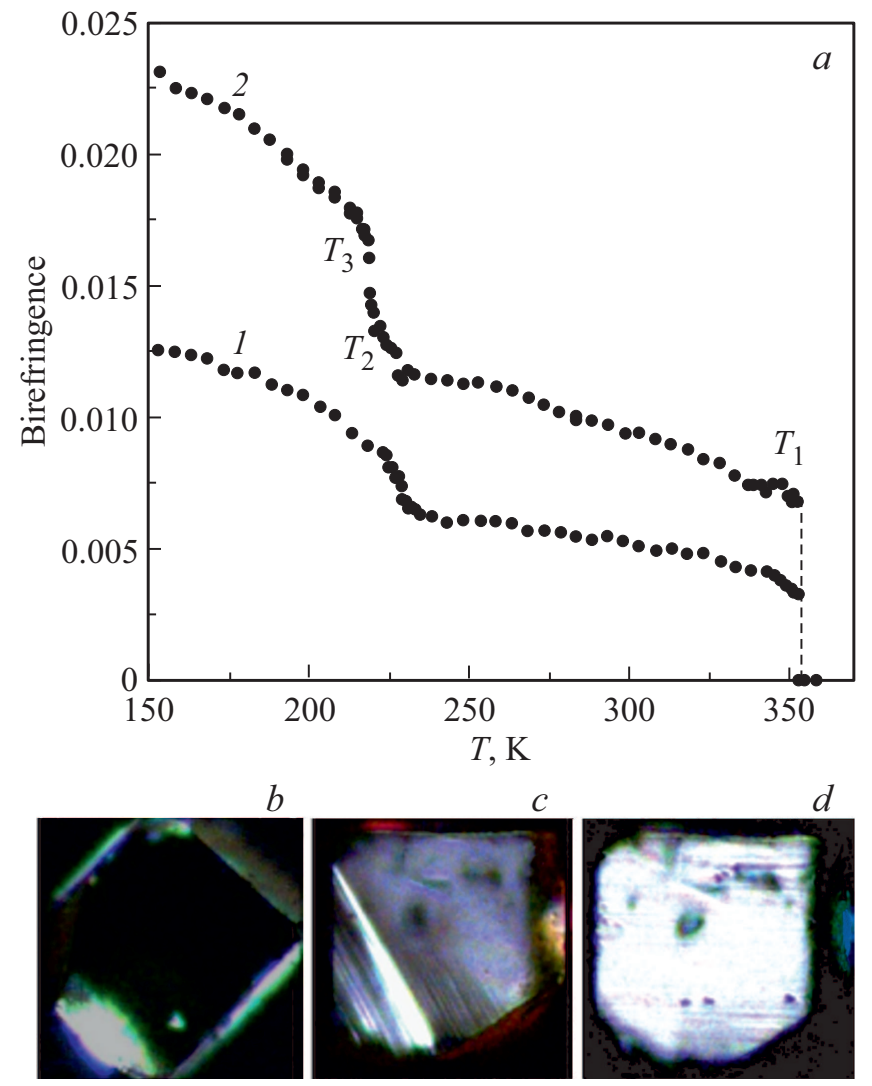

Рис. 5. Температурная зависимость двупреломления в пластинках (111) (1) и (110) (2) кубической фазы кристалла $\left(\mathrm{NH}_{4}\right)_{3} \mathrm{VOF}_{5}(a)$. Поляризационно-оптические наблюдения образцов (111) кубической структуры в фазах: $F m \overline{3} m(b)$, $\operatorname{Immm}(c)$ и $P \overline{1}(d)$.

упаковывался в инертной атмосфере гелия в фурнитуру с нагревателем. Измерения теплоемкости системы проводились в режимах непрерывных $(d T / d t=0.15 \mathrm{~K} / \mathrm{min})$ и дискретных $(\Delta T=2.5-3.0 \mathrm{~K})$ нагревов. Теплоемкость фурнитуры измерялась в отдельном эксперименте.

Температурная зависимость молярной изобарной теплоемкости $C_{p}(T)$ кристаллов $\left(\mathrm{NH}_{4}\right)_{3} \mathrm{VOF}_{5}$ характеризуется аномальным поведением при уточненных температурах фазовых переходов: $T_{1}=348.1 \pm 0.5 \mathrm{~K}$, $T_{2}=229.1 \pm 0.2 \mathrm{~K}$ и $T_{3}=218 \pm 1 \mathrm{~K}$ (рис. 6,a). Для определения интегральных характеристик выполнено разделение теплоемкости на регулярный вклад и аномальный вклад $\Delta C_{p}(T)$, связанный с последовательностью переходов. Для этого участки температурной зависимости теплоемкости вне области существования аномалий аппроксимировались комбинацией функций Дебая и Эйнштейна. Интегрированием температурной зависимости избыточной теплоемкости $\Delta C_{p}(T)$, получены величины изменения энтропии $\Delta S_{i}=\int\left(\Delta C_{p} / T\right) d T$, которыми характеризуются фазовые превращения в $\left(\mathrm{NH}_{4}\right)_{3} \mathrm{VOF}_{5}: \Delta S_{1}=5.4 \pm 0.5 \mathrm{~J} / \mathrm{mol} \cdot \mathrm{K}$ и $\Delta S_{2+3}=8 \pm 0.5 \mathrm{~J} / \mathrm{mol} \cdot \mathrm{K}$ (рис. $\left.6, b\right)$.
Измерения теплового расширения выполнены на дилатометре NETZSCH DIL 402C в температурном диапазоне $120-350 \mathrm{~K}$ в динамическом режиме со скоростью нагрева $\sim 3 \mathrm{~K} / \mathrm{min}$. Исследования проводились в потоке газообразного гелия при расходе $\sim 50 \mathrm{ml} \mathrm{min}^{-1}$. Для калибровки прибора и учета теплового расширения измерительной системы использовались эталоны из плавленого кварца [13]. Образцы $\left(\mathrm{NH}_{4}\right)_{3} \mathrm{VOF}_{5}$ были приготовлены в виде таблеток диаметром $\sim 4 \mathrm{~mm}$ и высотой $\sim 4-6 \mathrm{~mm}$ путем прессования при давлении $\sim 2 \mathrm{GPa}$.

В области температур фазовых переходов наблюдается аномальное поведение теплового расширения (рис. 7). Значительная величина скачка деформации $\delta\left(V / V_{0}\right)=1 \cdot 10^{-4} \mathrm{~K}$ характеризует переход при температуре $T_{1}$ как превращение первого рода. В низкотемпературной области более выражены анома-

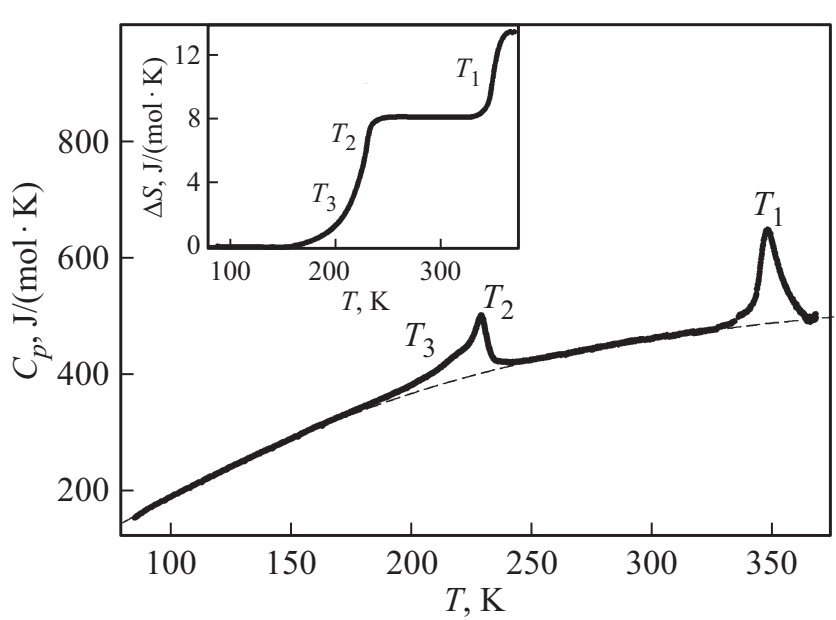

Рис. 6. Температурные зависимости теплоемкости и энтропии фазовых переходов (на вставке) в кристалле $\left(\mathrm{ND}_{4}\right)_{3} \mathrm{VOF}_{5}$ в широком интервале температур.

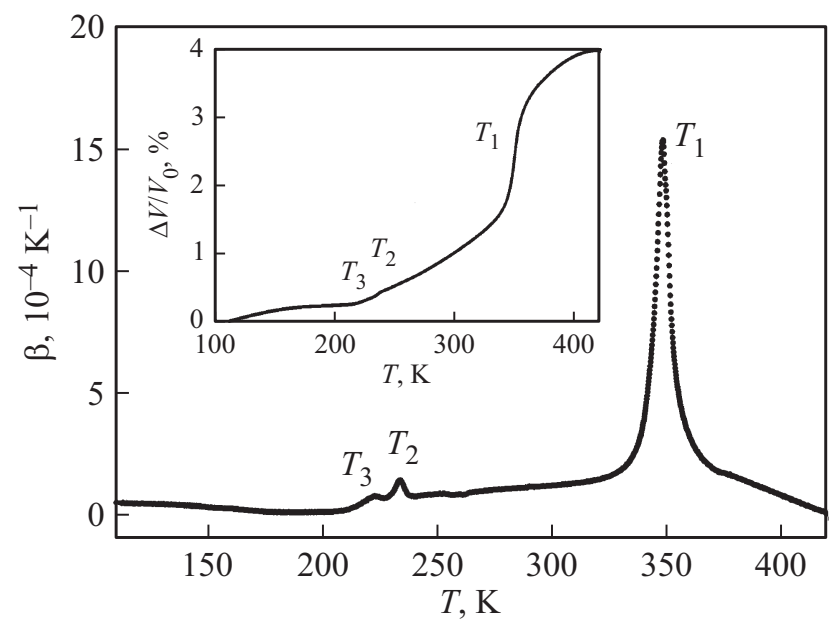

Рис. 7. Температурные зависимости коэффициента объемного расширения и деформации (на вставке) кристалла $\left(\mathrm{ND}_{4}\right)_{3} \mathrm{VOF}_{5}$. 
лии коэффициента объемного теплового расширения: $\Delta \beta\left(T_{2}\right)=0.62 \cdot 10^{-4} \mathrm{~K}^{-1}$ и $\Delta \beta\left(T_{3}\right)=0.24 \cdot 10^{-4} \mathrm{~K}^{-1}$.

Восприимчивость к гидростатическому давлению исследовалась методом дифференциально-термического анализа (ДТА). Поликристаллический образец массой $\sim 0.02 \mathrm{~g}$ упаковывался в медный контейнер, прикрепленный к одному из спаев термоэлектрического ДТА-элемента. Измерения проводились в камере высокого давления типа цилиндр-поршень, в качестве среды передающей гидростатическое давления использовалось силиконовое масло.

Вследствие температурных ограничений установки ДТА прямые измерения барической восприимчивости высокотемпературного фазового перехода были невозможны.

Температуры фазовых переходов $T_{2}$ и $T_{3}$ растут практически линейно при увеличении внешнего давления (рис. 8). Вследствие существенно разных величин барических коэффициентов $d T_{2} / d p=23 \pm 2 \mathrm{~K} / \mathrm{GPa}$ и $d T_{3} / d p=92 \pm 4 \mathrm{~K} / \mathrm{GPa}$ на фазовой диаграмме $T-p$ при давлении $p \approx 0.1 \mathrm{GPa}$ наблюдается тройная точка. Восприимчивость образовавшейся при высоком давлении фазовой границы Immm $\leftrightarrow P \overline{1}(P 1)$ характеризуется достаточной большой величиной барического коэффициента $d T^{*} / d p=80 \pm 1 \mathrm{~K} / \mathrm{GPa}$.

Исследования температурной зависимости диэлектрической проницаемости были выполнены с помощью измерителя иммитанса $\mathrm{E} 7-20$ на частоте $1 \mathrm{kHz}$ в интервале температур $100-320 \mathrm{~K}$. Образцы $\left(\mathrm{ND}_{4}\right)_{3} \mathrm{VOF}_{5}$ были приготовлены в виде таблеток диаметром $\sim 8 \mathrm{~mm}$ и высотой $\sim 1-2 \mathrm{~mm}$ путем прессования при давлении $\sim 2 \mathrm{GPa}$. В качестве электродов на образцы наносилось золото путем вакуумного напыления. Скорости изменения температуры в режимах нагрева и охлаждения составляли $\sim 0.7 \mathrm{~K} / \mathrm{min}$.

На температурных зависимостях диэлектрической проницаемости $\varepsilon(T)$ и диэлектрических потерь $\operatorname{tg} \delta(T)$

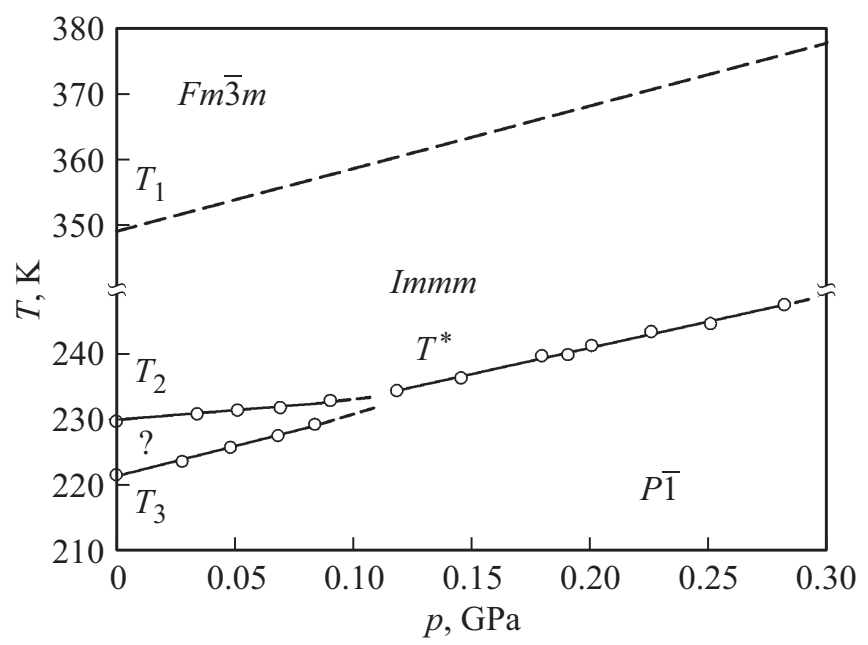

Рис. 8. Фазовая диаграмма температура-давление кристалла $\left(\mathrm{ND}_{4}\right)_{3} \mathrm{VOF}_{5}$. Линия раздела фаз $F m \overline{3} m$ и Immm (штриховая линия) восстановлена на основании расчета $\left(d T_{1} / d p\right)_{\text {calc }}$.

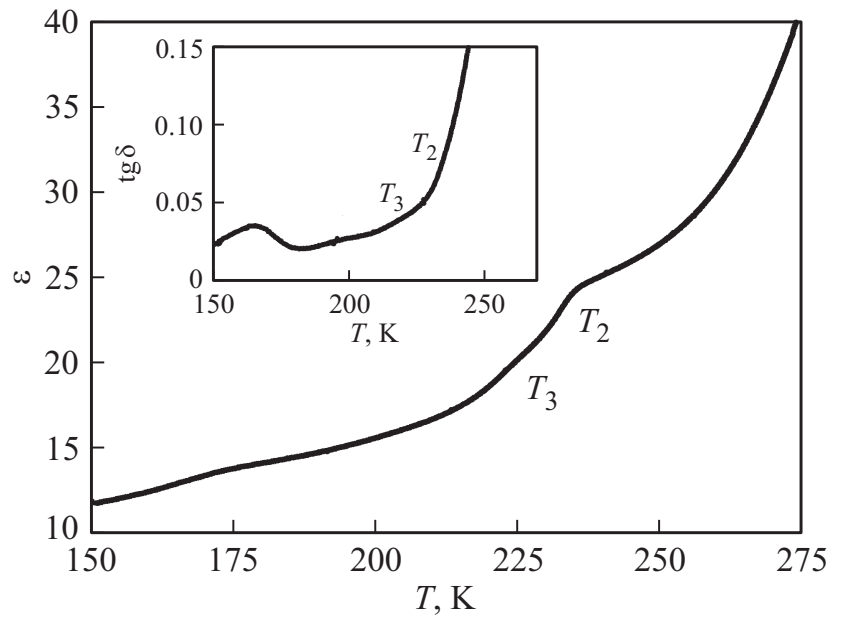

Рис. 9. Температурные зависимости диэлектрической проницаемости $\varepsilon(T)$ и диэлектрических потерь $\operatorname{tg} \delta(T)$ (на вставке) в кристаллах $\left(\mathrm{ND}_{4}\right)_{3} \mathrm{VOF}_{5}$.

(рис. 9) в области фазовых переходов при температурах $T_{2}$ и $T_{3}$ происходит ступенчатое увеличение диэлектрической проницаемости $\Delta \varepsilon_{1} \approx \Delta \varepsilon_{2} \approx 2$. Такое поведение $\varepsilon(T)$ является характерным для фазовых переходов, имеющих несегнетоэлектрическую природу. Аномальное поведение в области высокотемпературного фазового перехода сопровождается значительным ростом диэлектрической проницаемости, причиной которого является существенное увеличение диэлектрических потерь в области высоких температур $T>270 \mathrm{~K}$ (рис. 9, $b$ ).

\section{3. Обсуждение результатов}

Парафаза исследуемого соединения $\left(\mathrm{NH}_{4}\right)_{3} \mathrm{VOF}_{5}$ изоструктурна эльпасолиту $(F m \overline{3} m, Z=4)[14,15]$. Исходя из анализа структурных данных, атомы F и O занимают одну позицию $24 e$ с заселенностью 5/6 и 1/6 (табл. 2). Их совместный тепловой параметр уточнялся в анизотропном приближении. Однако у электронной плотности были обнаружены дополнительные максимумы в позиции $48 h$, соответствующие атомам $\mathrm{F}(\mathrm{O}), \mathrm{O} 1(\mathrm{~F} 1)$ и $\mathrm{O} 2(\mathrm{~F} 2)$. Поэтому в финальном уточнении были использованы две позиции O1/F1 и O2/F2. На заселенности позиций наложены ограничения посредством линейных уравнений, чтобы результирующая формула оказалась $\left(\mathrm{NH}_{4}\right)_{3} \mathrm{VOF}_{5}$.

$\mathrm{B}$ результате фазового перехода структура $\left(\mathrm{ND}_{4}\right)_{3} \mathrm{VOF}_{5}$ меняется на ромбическую (пр.гр. Immm) и имеет параметры ячейки, сходные с кристаллом $\left(\mathrm{NH}_{4}\right)_{3} \mathrm{VO}_{2} \mathrm{~F}_{4}$ [6]. Вследствие сильного двойникования структура кристалла $\left(\mathrm{NH}_{4}\right)_{3} \mathrm{VO}_{2} \mathrm{~F}_{4}$ не была полностью определена даже для монокристалла $[6,8]$, в результате чего модель разупорядочения одного из двух независимых полиэдров $\left[\mathrm{VO}_{2} \mathrm{~F}_{4}\right]^{3-}$ достоверно не установлена. Модель разупорядочения $\left(\mathrm{NH}_{4}\right)_{3} \mathrm{VOF}_{5}$ также невозможно установить из проведенных рентгеновских исследований. 
Таблица 2. Координаты атомов, заселенности позиций $p$, а также изотропный $B_{i s o}$ и анизотропный $U_{i j}$ тепловые параметры кристалла $\left(\mathrm{NH}_{4}\right)_{3} \mathrm{VOF}_{5}$ при $T=383 \mathrm{~K}$.

\begin{tabular}{c|c|c|c|c|c}
\hline Атом & $x$ & $y$ & $z$ & $p$ & $B_{i s o} / U_{i j}$ \\
\hline $\mathrm{V}$ & 0 & 0 & 0 & 1 & $5.8(2)$ \\
$\mathrm{N} 1$ & $1 / 2$ & $1 / 2$ & $1 / 2$ & 1 & $12.4(4)$ \\
$\mathrm{H} 1$ & 0.55 & 0.55 & 0.55 & 0.5 & $7.1(3)$ \\
$\mathrm{N} 2$ & $1 / 4$ & $1 / 4$ & $1 / 4$ & 1 & 5 \\
$\mathrm{H} 2$ & 0.198 & 0.198 & 0.198 & 1 & $U_{11}=0.011(5)$ \\
$\mathrm{F} 1$ & $0.2098(4)$ & 0 & 0 & $0.691(8)$ & $U_{22}=U_{33}=0.173(5)$ \\
& & & & & $U_{11}=0.011(5)$ \\
O1 & $0.2098(4)$ & 0 & 0 & $0.138(2)$ & $U_{22}=U_{33}=0.173(5)$ \\
$\mathrm{F} 2$ & $0.366(2)$ & $0.366(2)$ & 0 & $0.071(4)$ & $5.0(8)$ \\
O2 & $0.366(2)$ & $0.366(2)$ & 0 & $0.0143(8)$ & $5.0(8)$
\end{tabular}

Температурная зависимость объема элементарной ячейки характеризуется значительным скачком объема $\Delta V_{c} \approx 13.8 \AA$, который наблюдается при фазовом превращении при $T_{1}$ (рис. 10). Таким образом, высокотемпературное превращение является фазовым переходом первого рода. Информация об изменении энтропии $\Delta S_{1}$ высокотемпературного фазового перехода, полученная из проведенных теплофизических экспериментов, позволяет оценить наклон фазовой границы $F m \overline{3} m \leftrightarrow I m m m$. Величина барического коэффициента определена из уравнения Клапейрона-Клаузиуса: $d T / d p_{1}=\left(\Delta V N_{A}\right) /(Z \Delta S) \approx 128 \mathrm{~K} / \mathrm{GPa}$, где $\Delta V$ - изменение объема кубической ячейки при фазовом переходе, $N_{A}$ - число Авогадро, $Z=4$ - число формульных единиц в ячейке.

В результате исследования температурной зависимости $C_{p}(T)$ установлено наличие избыточной теп-

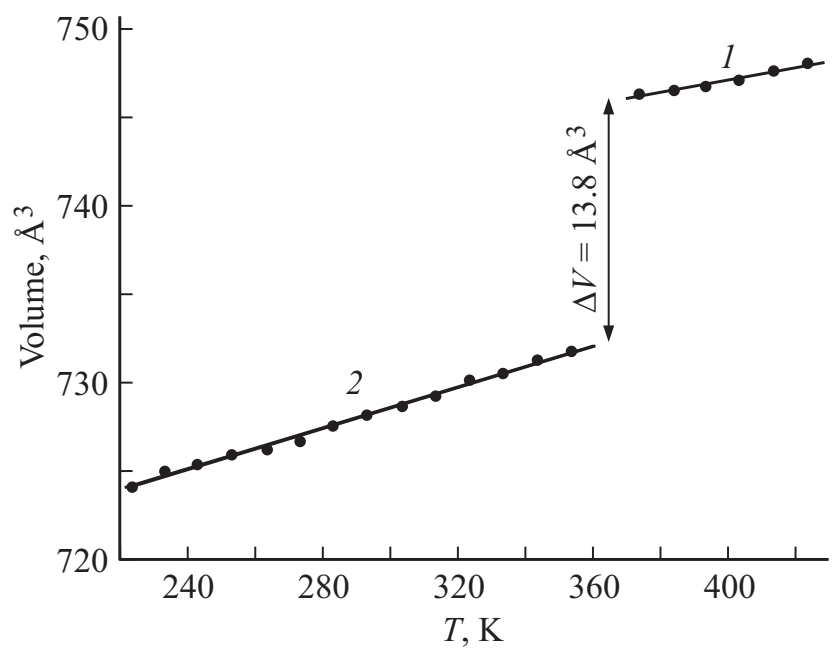

Рис. 10. Температурные зависимости объема ячеек $V_{\text {сиь }}(1)$ и $V_{\text {otrh }} 2 / 3$ (2).

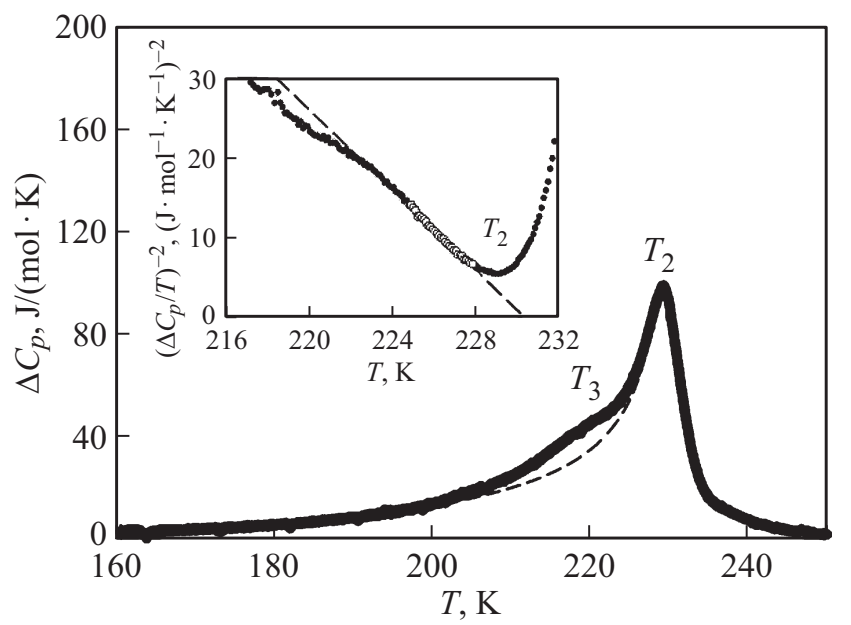

Рис. 11. Температурные зависимости избыточной теплоемкости и квадрата обратной ее величины (на вставке) в окрестности $T_{2}$.

лоемкости в довольно широком интервале температуры ниже $T_{2}$ (рис. 11,a), что позволило выполнить анализ фазового превращения Iтmm $\leftrightarrow$ моноклинная фаза в рамках термодинамической теории Ландау. В соответствии с [16], квадрат обратной избыточной теплоемкости является линейной функцией температуры: $\left(\Delta C_{p} / T\right)^{-2}=\left[2\left(B^{2}-3 A^{\prime} C\right)^{0.5} / A_{T}^{2}\right]^{2}+12 C$ $\times\left(T_{o}-T\right) / A_{T}^{3}$, где величины $A=A_{T}\left(T_{0}-T_{C}\right)+A_{T}\left(T-T_{0}\right)$ $=A^{\prime}+A_{T}\left(T-T_{0}\right), \quad B \quad$ и $C$ являются коэффициентами термодинамического потенциала: $\Delta \Phi(p, T, \eta)=$ $=A \eta^{2}+B \eta^{4}+C \eta^{6} \quad\left(\eta-\right.$ параметр перехода, $T_{C}-$ температура Кюри, $T_{0}$ - температура фазового перехода). Температурное поведение величины квадрата обратной избыточной теплоемкости $\left(\mathrm{NH}_{4}\right)_{3} \mathrm{VOF}_{5}$ ниже температуры $T_{2}$ достаточно хорошо описывается линейной функцией (рис. 11, b). Рассчитанные параметры 
Таблица 3. Термодинамические параметры фазовых переходов в оксифторидах $\left(\mathrm{NH}_{4}\right)_{3} \mathrm{VOF}_{5}$ и $\left(\mathrm{NH}_{4}\right)_{3} \mathrm{VO}_{2} \mathrm{~F}_{4}$.

\begin{tabular}{l|c|c}
\hline \multicolumn{1}{c|}{ Параметр } & $\left(\mathrm{NH}_{4}\right)_{3} \mathrm{VOF}_{5}$ & $\left(\mathrm{NH}_{4}\right)_{3} \mathrm{VO}_{2} \mathrm{~F}_{4}[9]$ \\
\hline$T_{1}, \mathrm{~K}$ & $348.1 \pm 0.5$ & $438 \pm 1$ \\
$\Delta S_{1}, \mathrm{~J} / \mathrm{mol} \cdot \mathrm{K}$ & $5.4 \pm 0.5$ & $9.7 \pm 1.0$ \\
$\left(d T_{1} / d p\right)_{\mathrm{calc}}, \mathrm{K} \cdot \mathrm{GPa}^{-1}$ & $115 \pm 10$ & - \\
$T^{\prime}, \mathrm{K}$ & & $244.0 \pm 0.2$ \\
$\Delta S^{\prime}, \mathrm{J} / \mathrm{mol} \cdot \mathrm{K}$ & $229.1 \pm 0.2$ & $0.18 \pm 0.04$ \\
$T_{2}, \mathrm{~K}$ & -0.6 & $210.2 \pm 0.2$ \\
$\left(A_{T}^{2} / B\right)_{T_{2}}, \mathrm{~J} / \mathrm{mol} \cdot \mathrm{K}^{2}$ & 4.6 & -0.4 \\
$\left(A_{T}^{3} / C\right)_{T_{2}}, \mathrm{~J}^{2} / \mathrm{mol}^{2} \cdot \mathrm{K}^{3}$ & 3.2 & 1.1 \\
$T_{2}-T_{C_{2}}, \mathrm{~K}$ & -0.13 & 1.4 \\
$N_{2}$ & 7.6 & -0.1 \\
$\Delta S_{2}, \mathrm{~J} / \mathrm{mol} \cdot \mathrm{K}$ & $23 \pm 2$ & $-27 \pm 3$ \\
$\left(d T_{2} / d p\right)_{\exp }, \mathrm{K} \cdot \mathrm{GPa}^{-1}$ & $218 \pm 1$ & $205.1 \pm 0.2$ \\
$T_{3}, \mathrm{~K}$ & - & -1.3 \\
$\left(A_{T}^{2} / B\right)_{T_{3}}, \mathrm{~J} / \mathrm{mol}^{2} \cdot \mathrm{K}^{2}$ & - & 2.2 \\
$\left(A_{T}^{3} / C\right)_{T_{3}}, \mathrm{~J}^{2} / \mathrm{mol}^{2} \cdot \mathrm{K}^{3}$ & - & 0.4 \\
$T_{3}-T_{C_{3}}, \mathrm{~K}$ & - & -0.05 \\
$N_{3}$ & $0.4 \pm 0.06$ & $0.80 \pm 0.12$ \\
$\Delta S_{3}, \mathrm{~J} / \mathrm{mol} \cdot \mathrm{K}$ & $92 \pm 4$ & $-25 \pm 3$ \\
$\left(d T_{3} / d p\right)_{\exp }, \mathrm{K} \cdot \mathrm{GPa}^{-1}$ & &
\end{tabular}

феноменологической модели фазового перехода при $T_{2}$ (табл. 3) показывают, что в кристаллах $\left(\mathrm{NH}_{4}\right)_{3} \mathrm{VOF}_{5}$ превращение Immm $\leftrightarrow$ моноклинная фаза находится дальше от трикритической точки $\left(T_{i}-T_{C i}=0\right.$ и $N=0$, где $N= \pm\left[B^{2} /\left(3 A_{T} C T_{0}\right)\right]^{-0.5}-$ степень близости перехода к трикритической точке), чем фазовый переход Immm $\leftrightarrow P 112 / m$, который наблюдается при температуpe $T_{3}$ в $\left(\mathrm{NH}_{4}\right)_{3} \mathrm{VO}_{2} \mathrm{~F}_{4}$ [9]. Экстраполяция зависимости $\left(\Delta C_{p} / T\right)^{-2}(T)$, полученной из феноменологической модели, позволяет разделить энергетические вклады фазовых переходов при температурах $T_{2}$ и $T_{3}$ (рис. $11, a$ ), и оценить величину индивидуальных изменений энтропии $\Delta S_{2}$ и $\Delta S_{3}$ (табл. 3).

Совместный анализ экспериментальных данных о теплоемкости и тепловом расширении позволил рассчитать барическую восприимчивость кристаллических фаз, реализующихся в $\left(\mathrm{NH}_{4}\right)_{3} \mathrm{VOF}_{5}$. С использованием величин скачков энтропии $\Delta S_{1} \approx 2.8 \mathrm{~J} / \mathrm{mol} \cdot \mathrm{K}$ (рис. $\left.7, b\right)$ и деформации $\delta\left(\Delta V / V_{0}\right)=0.01$ (рис. $\left.8, a\right)$ при $T_{1}$ из уравнения Клапейрона-Клаузиуса был рассчитан соответствующий барический коэффициент $\left(d T_{1} / d p\right)_{\text {calc }} \approx 115 \mathrm{~K} / \mathrm{GPa}$. Близкие величины $d T_{1} / d p$, полученные из рентгеновских и дилатометрических данных, свидетельствуют о надежности результатов независимых экспериментов.

Для низкотемпературных фазовых переходов барические коэффициенты $\left(d T_{2} / d p\right)_{\text {calc }} \approx 26 \mathrm{~K} / \mathrm{GPa} \quad$ и $\left(d T_{3} / d p\right)_{\text {calc }} \approx 120 \mathrm{~K} / \mathrm{GPa}$ определены в рамках соотношения Эренфеста $\Delta C_{p}=\Delta \beta T_{i} /\left(d T_{i} / d p\right)_{p=0}[17]$. Несмотря на сложности определения индивидуальных параметров низкотемпературных фазовых переходов согласие между экспериментальными и рассчитанными бариче- скими коэффициентами можно считать удовлетворительным (табл. 3).

Замещение центрального атома, $\mathrm{V}^{4+} \rightarrow \mathrm{V}^{5+}$, привело к увеличению объема элементарной ячейки $\left(\mathrm{NH}_{4}\right)_{3} \mathrm{VOF}_{5}\left(V_{c}=746 \AA^{3}\right)$ по сравнению с $\left(\mathrm{NH}_{4}\right)_{3} \mathrm{VO}_{2} \mathrm{~F}_{4}$ $\left(V_{c}=735 \AA^{3}[5]\right)$, которое можно рассматривать как связанное с уменьшением химического давления. В соответствии с положительным знаком барического коэффициента $d T_{1} / d p$ для $\left(\mathrm{NH}_{4}\right)_{3} \mathrm{VOF}_{5}$ это должно приводить к росту температуры устойчивости кубической фазы в $\left(\mathrm{NH}_{4}\right)_{3} \mathrm{VO}_{2} \mathrm{~F}_{4}$, что и наблюдалось в экспериментах (табл. 3). Оцененная величина избыточного химического давления составляет $\Delta p=0.8 \mathrm{GPa}$.

В рамках структурной модели [6] исходная кубическая фаза $\left(\mathrm{NH}_{4}\right)_{3} \mathrm{VOF}_{5}$ характеризуется разупорядочением как фторкислородного аниона $\left[\mathrm{VOF}_{5}\right]^{3-}$, так и аммонийных катионов, которое должно приводить к большой величине энтропии при фазовых переходах. Однако экспериментально установленное значение $\sum_{i} S_{i} \approx 14 \mathrm{~J} / \mathrm{mol} \cdot \mathrm{K}$ оказалось относительно небольшим, хотя и характерным для процессов порядок-беспорядок. Аналогичная ситуация наблюдалась при исследованиях изоструктурного кристалла $\left(\mathrm{NH}_{4}\right)_{3} \mathrm{VO}_{2} \mathrm{~F}_{4}$, в котором предполагалось еще большее разупорядочение структурных элементов [6]. Однако суммарная установленная из теплофизических исследований [9], величина изменения энтропии, связанная с последовательными превращениями, оказалась ненамного превышающей аналогичную величину для $\left(\mathrm{NH}_{4}\right)_{3} \mathrm{VOF}_{5}$.

\section{4. Заключение}

Выращены однофазные кристаллы аммонийного оксопентафторванадата (IV), $\left(\mathrm{NH}_{4}\right)_{3} \mathrm{VOF}_{5}$. Анализ широкого спектра проведенных исследований (оптических, теплофизических, структурных, диэлектрических) совместно с данными, полученными ранее для изоструктурного соединения $\left(\mathrm{NH}_{4}\right)_{3} \mathrm{VO}_{2} \mathrm{~F}_{4}[6,9]$, позволил установить особенности физических свойств оксифторидов, содержащих ванадий разной валентности $\mathrm{IV}(\mathrm{V})$.

Уменьшение химического давления в $\left(\mathrm{NH}_{4}\right)_{3} \mathrm{VOF}_{5}$, вызванное увеличением объема элементарной ячейки по сравнению с $\left(\mathrm{NH}_{4}\right)_{3} \mathrm{VO}_{2} \mathrm{~F}_{4}$, сопровождается:

1) значительным изменением температур фазовых переходов и интервалов устойчивости исходной и искаженных фаз;

2) уменьшением энтропий фазовых переходов, что качественно согласуется с моделями разупорядочения кубической структуры [6];

3) выклиниванием промежуточной моноклинной фазы в $\left(\mathrm{NH}_{4}\right)_{3} \mathrm{VOF}_{5}$ при относительно низком давлении;

4) сохранением сегнетоэластической (несегнетоэлектрической) природы структурных искажений;

5) незначительным изменением степени близости фазовых переходов к трикритической точке. 


\section{Благодарности}

Авторы выражают глубокую признательность А.С. Романченко за любезно предоставленные результаты, полученные методом рентгеновской фотоэлектронной спектроскопии. Рентгенографические и дилатометрические данные получены с использованием оборудования Красноярского регионального центра коллективного пользования ФИЦ КНЦ СО РАН.

\section{Финансирование}

Исследования выполнены при финансовой поддержке Российского фонда фундаментальных исследований, Правительства Красноярского края, Красноярского краевого фонда науки в рамках научного проекта: „Влияние дейтерирования на ориентационное упорядочение и фазовые переходы в аммонийных фторкислородных ванадатах“" № 18-42-243003.

\section{Конфликт интересов}

Авторы заявляют, что у них нет конфликта интересов.

\section{Список литературы}

[1] G. Pausewang, K. Dehnicke, Z. Anorg. Allg. Chem. 369, 265 (1969).

[2] I.N. Flerov, M.V. Gorev, K.S. Aleksandrov, A. Tressaud, J. Grannec, M. Couzi. Mater. Sci. Eng. 24, 81 (1998).

[3] R. Gautier, M.D. Donakowski, K.R. Poeppelmeier. J. Solid State Chem. 195, 132 (2012).

[4] S.J. Patwe, A.S. Nagabhusan, K.G. Girija, C.G. Sivan Pillai, A.K. Tyagi. J. Mater. Res. 25, 1251 (2010).

[5] M. Leimkühler, R.J. Mattes. Solid State Chem. 65, 260 (1986).

[6] A.A. Udovenko, E.I. Pogoreltsev, Y.V. Marchenko, N.M. Laptash. Acta Cryst. B73, 1 (2017).

[7] Yu.V. Gerasimova, A.S. Oreshonkov, N.M. Laptash, A.N. Vtyurin, A.S. Krylov, N.P. Shestakov, A.A. Ershov, A.G. Kocharova. Spectrochim. Acta A 176, 106 (2017).

[8] С.В. Мельникова, А.Г. Кочарова. ФТТ 51, 3, 562 (2009).

[9] V.D. Fokina, M.V. Gorev, A.G. Kocharova, E.I. Pogoreltsev, I.N. Flerov. Solid State Sci. 11, 836 (2009).

[10] Е.В. Богданов, Е.И. Погорельцев, М.В. Горев, М.С. Молокеев, И.Н. Флеров. ФТТ 61, 2, 330 (2019).

[11] Bruker AXS TOPAS V4: General profile and structure analysis software for powder diffraction data.User's Manual, Bruker AXS, Karlsruhe, Germany (2008).

[12] В.И. Михеев. Рентгенометрический определитель минералов. Геология и охрана недр, М: (1957). 867 с.

[13] М.В. Горев, Е.В. Богданов, И.Н. Флёров, А.Г. Кочарова, Н.М. Лапташ. ФТТ 52, 1, 156 (2010).

[14] М.С. Молокеев, С.В. Мисюль. ФТТ 54, 1, 144 (2012).

[15] В.Д. Фокина, И.Н. Флёров, М.С. Молокеев, Е.И. Погорельцев, Е.В. Богданов, А.С. Крылов, А.Ф. Бовина, В.Н. Воронов, Н.М. Лапташ. ФТТ 50, 11, 2084 (2008).

[16] К.С. Александров, И.Н. Флёров. ФТТ 21, 327 (1979).

[17] Н. Парсонидж, Л. Стейвли. Беспорядок в кристаллах. Мир, М. (1982). 436 c.

Редактор Е.Ю. Флегонтова 